tion for parallel analysis of sets of target sequences. The method employs arrayed primers that bind specific padlock probes and initiate rolling-circle replication. A localized, linear concatemer is generated that is detected by fluorescence microscopy in a microarray format. Recently we have shown that padlock probes can also be ligated on RNA template with good sequence discrimination. Therefore, this method may also be suitable for analysis of RNA expression. The ability of padlock probes to detect RNA sequence variation may facilitate the analysis of closely related genes and alternatively spliced transcripts, which have been difficult to study with cDNA-based expression arrays. The inherent signal amplification will aid in quantitation of low-abundance RNA, and may expand the use of expression arrays by permitting the study of small cell populations such as developing tissues and solid tumours

Iyer, Vishy

\section{Genome-wide maps of DNA-protein interactions using a yeast ORF and intergenic microarray}

\section{Vishwanath R. Iyer' ${ }^{1}$ Christine Horak2, Laurent Kuras³, Peter Kosa ${ }^{3}$, Charles Scafe ${ }^{4}$, David Botstein ${ }^{4}$, Kevin Struhl ${ }^{3}$, Michael Snyder ${ }^{2}$ \& Patrick O. Brown \\ ${ }^{1}$ Department of Biochemistry and Howard Hughes M edical Institute, Stanford University M edical Center, Stanford, California 94305, USA \\ ${ }^{2}$ Department of M olecular, Cellular \& Development Biology, Yale University, New Haven, Connecticut, USA \\ ${ }^{3}$ Department of Biological Chemistry and M olecular Pharmacology, Harvard M edical School, Boston, M assachusetts, USA \\ ${ }^{4}$ Department of Genetics, Stanford University, Stanford, California 94305, USA}

We have made a DNA microarray that includes not only all the open reading frames (ORFs) and other features in the yeast genome, but also all the intergenic regions. We are using this as a tool to construct genome-wide maps of DNA-protein interactions for proteins that interact directly or indirectly with DNA or chromatin in vivo. Proteins are crosslinked to DNA in vivo using formaldehyde and the crosslinked DNA is extracted and sheared. DNA specifically associated with any protein of interest is immunoprecipitated using a specific antibody against the protein or an epitope tag that may be fused to the protein. The selected DNA, representing loci that the protein interacts with in vivo, can be identified by fluorescently labelling it and hybridizing it to the microarray along with an appropriate reference probe. This approach is being used to map the genome-wide interactions of sequence-specific DNA binding proteins, components of the transcription machinery and chromatin components, under a variety of conditions.

We have analysed the interaction with the genome of SWI4, a sequence specific DNA binding activator that regulates genes expressed in the G1/S phase of the cell cycle. We detected specific interactions of SWI4 with the promoters of the HO, CLN1, CLN2, PCL1 and other G1/S specific genes. A majority, but not all, of the sites that SWI4 appears to interact with in vivo are in the promoters of genes that are G1/S specific. Initiation of transcription requires the specific binding of TATA-box binding protein (TBP) to the core promoter and the enhancement of this is believed to be a key step in the activation of transcription. Using the intergenic microarrays, we found increased association of TBP with the promoters of genes induced by heat shock, 15 minutes after yeast cells were heat shocked. This supports the idea that recruitment of TBP to these promoters is increased upon heat shock and is an important step in the process of transcriptional activation of these genes.

Comparison of the sites of actual interaction of a protein in vivo with its predicted binding sites is likely to give us a better idea of the sequence determinants for DNA interactions. Since these microarrays represent nearly every locus in the yeast genome, they can also be used to identify novel transcripts in the intergenic regions that may not have been predicted by the sequence annotation of the genome.
Jennings, Ezra

\section{Combinatorial negative regulation of glu- cose-responsive genes in yeast}

\author{
Ezra G. Jennings ${ }^{1,2}$, Frank C.P. Holstege ${ }^{1}$, John J. W yrick ${ }^{1,2}$ \\ \& Richard A. Young ${ }^{1,2}$ \\ ${ }^{1}$ W hitehead Institute for Biomedical Research, Nine Cambridge Center, \\ Cambridge, M assachusetts 02142, USA \\ 2Department of Biology, M assachusetts Institute of Technology, Cambridge, \\ M assachusetts 02139, USA
}

Glucose is the preferred carbon source for cells, and the molecular machinery responsible for glucose uptake and metabolism is well understood. In contrast, how cells reprogram genome-wide expression when they encounter glucose is not known. We used genome-wide expression analysis to elucidate the global regulatory response to glucose and to investigate how negative regulators participate in glucose repression. Our results reveal that $6 \%$ of yeast genes are induced and $8 \%$ are repressed by exposure to glucose. We find that the negative regulatory response is largely effected through the combined action of two negative regulators that act on TATA-binding protein (NC2 and Mot1), components of the RNA polymerase II holoenzyme (Srb10 and Sin4), a factor recruited to promoters by sequence-specific DNA-binding proteins (Tup1) and histones. Although previous studies have established that proper expression of many genes depends on the combined action of multiple positive regulators, these results provide evidence for combinatorial regulation of a substantial portion of the genome through highly conserved negative regulators.

Jia, Libin

\section{Gene expression profiling of human bone marrow stromal cells with FGFR-3 mutation (K650M) using CDNA microarrays}

\author{
Libin Jia ${ }^{1}$, Michael Bittner', Yidong Chen ${ }^{1}$, Kris Gooden ${ }^{1}$, Yuan \\ Jiang' ${ }^{1}$, Jeffery Trent', Erica Keen ${ }^{2}$, Anna R. DeRubeis ${ }^{3}$, Sergei \\ Kuznetsov ${ }^{3}$, Pamela Robey ${ }^{3}$, M arian Young ${ }^{3}$, John Powell ${ }^{4}$, Liming \\ Yang $^{4}$, Robert Hotchkiss ${ }^{5} \&$ Claire Francomano $^{1}$ \\ ${ }^{1} \mathrm{NHGRI}, \mathrm{NIH}$, Bethesda, M aryland, USA \\ ${ }^{3} \mathrm{NIDR}, \mathrm{NIH}$, Bethesda, M aryland, USA \\ ${ }^{2}$ Yale University, New Haven, Connecticut, USA \\ ${ }^{4} \mathrm{CIT}$, NIH, Bethesda, M aryland, USA \\ ${ }^{5}$ Hospital for Special Surgery, New York, New York, USA
}

Microarrays of cDNA provide a powerful tool for studying these complex phenomena. We used a high-density microarray of 1,400 cDNA elements to search for differences in gene expression associated with fibroblast growth factor receptor 3 (FGFR-3) K650M mutation. In this system, fluorescent probes for hybridisation were derived from two sources of human bone marrow stromal cellular mRNA (H54 and FGFR-3 K650M), which were labelled with different fluors to provide a direct and internally controlled comparison of the mRNA corresponding to each arrayed gene. We analysed the fluorescence signals representing hybridisation to each arrayed gene to determine the relative abundance in the two samples of mRNAs corresponding to each gene. There are at least 20 genes which are highly expressed in the control cells or FGFR-3 K650M mutation cells. For the gene expression level, 6 of these interesting genes were confirmed by northern blot analysis. Previously unrecognised alterations in the expression of specific genes provide leads for further investigation of the genetic basis of the dysplasia phenotype of FGFR-3 mutant cells. 